\title{
Teaching and Learning Music Theory in the Age of AI and Mobile Technologies
}

\author{
Eddy K. M. Chong \\ National Institute of Education, Nanyang Technological University, Singapore
}

\begin{abstract}
Mobile technologies coupled with the power of artificial intelligence offer many affordances for educators and learners alike. This paper presents a case of a music mobile app, Harmonia-on-the-Go, created for the purpose of teaching and learning an area in music theory that deals with chord grammar. As a kind of personal tutoring tool, the app generates automated feedback that reinforces the harmonic theory to be learnt. Student users can practise creating chord progressions anytime, anywhere, with immediate feedback given. If registered as a user, they can seek supplementary asynchronous feedback from the music teacher; learning analytics will also be generated for their self-monitoring of their own learning. For the teacher, the app reduces mundane marking but more invaluably, it frees up time for them to focus on monitoring students' learning as reflected in the learning analytics generated. They can in turn offer remedial teaching if necessary. The "Consult" function in the app also gives the teacher an additional platform, outside of class, to address students' questions. The article presents some brief observations from the pilot testing of the app, conducted with fifty high school students. It ends by articulating how this app can potentially transform the teaching and learning of music theory.
\end{abstract}

\section{Introduction}

The past decade saw the exciting confluence of two technological developments for educators - mobile technology and artificial intelligence (AI). After more than half a century of existence, AI saw a surge in interests in the present decade [1]. In the field of education, it has even been touted as "a game changer in education" [2]. Combined with learning analytics, AI can indeed be harnessed to create powerful personalised tutoring tools, especially when selfregulated learning is desired.

Mobile technology has also been around for quite some time, albeit for much less compared to AI.
Again, its advent has attracted much interests amongst educators [3-5]. With the increasing ubiquity of personal mobiles supporting the eager adoption by many educators [6], we are clearly presented with the opportunity to take advantage of what an age of mobile learning offers.

It was with this technological landscape in mind that a music tutoring mobile app, Harmonia-on-theGo, was developed and piloted - for both Android and iOS platforms. The intention was to create a "personal tutor" for students to reinforce their learning of harmonic theory through convenient and unrestricted self-practice, with immediate feedback provided. For the teacher, this mobile app serves as a kind of teacher-aide, freeing up time spent on mundane marking in order to better monitor students' learning and address any teaching-remedial needs.

\section{Teaching and learning of music theory}

One of the foundational studies in music is music theory. Insofar as music is a language, there is a need to understand its grammar. But, like all languages, the musical language has evolved over time and diversified in myriad ways with the proliferation of musical styles. Fortunately, there is a well-recognized common-practice period during which the foundation for many western musical styles was established. It is this common-practice harmonic language that is the focus of study in most western music curriculum; the present Harmonia-on-the-Go app deals with this harmonic grammar.

In most music theory courses, the general harmonic principles and more specific voice-leading rules (that is, grammatical rules that govern the rendition of musical lines in a particular harmonic context) are taught and students then apply them in music-writing exercises (e.g. harmonization of given melodies), music analysis, and composing. Whilst the learning output in these applicational exercises may differ in their mode of demonstration, the underlying harmonic understanding can be gleaned from the use of chord symbols insofar as the series of chord 
symbols betrays the students' harmonic understanding. The teacher can then assess students' understanding by evaluating this abstract representation.

There is one downside, however. The abstract nature of the music-symbol system does mean that the evaluation process can be mundane and musically unexciting. Yet, whilst analytical and compositional demonstrations may be more musically-rewarding for teachers to evaluate, they place additional demands on the novice learner to demonstrate their understanding via these modes. As such, there is a place for practice involving just abstract chord symbols. Given such pedagogical considerations, automation of the evaluation process would be a welcome for teachers, and, if feedback can be automatically generated, it would be an even greater boon.

\section{Developing the music AI}

\subsection{Choice of a rule-based AI approach}

In designing the music app to provide feedback that relates to the harmonic theory of classical music, a rule-based AI system was chosen rather than one based on statistical modeling or other approaches to machine learning. For the purpose at hand, not only must the evaluation be based on the harmonic theory in question, the feedback needs to inform the user in similar technical terms too. In other words, the AI system cannot be a "blackbox" but must have its "musical thinking" explicitly based on the intended harmonic theory.

To be clear, there are numerous music apps in both the iOS App Store and Google Play Store that allow users to create chord progressions, but most of them operate with a built-in system to constrain the chord selection so that the musical possibilities are limited for easier control to ensure acceptable results. A few provides technical feedback but these tend to be only of the very rudimentary kind. Chordrill (iOS), for example, evaluates users' identification of single chords. Chorder (iOS) would go so far as to indicate whether the chosen chords fit into the chosen scale. Neither app gets to the level of evaluating the chordprogression grammar.

By far, as far as the author is able to ascertain from a good search in both the App and Play stores, the most sophisticated feedback is offered by Tonal Harmony Analysis (iOS and Android). Users are provided with a set of chords in four-voice layout to select from. Once they have chosen a pair of chords, they are shown all possible ways to connect them in four-voice format as well as all possible erroneous ones, with the error(s) indicated. Users can then choose a particular correct pair and save onto the worksheet. In this manner, the user can gradually build the harmonic progression chord-by-chord. The music app in this case enables the addition of the new chord but it does not evaluate the entire chord progression.

\subsection{Chord representation}

For the music learning through Harmonia-on-the$G o$ to be most generalizable, it was decided that the creating of chord progression will involve abstract chord symbols instead of specifically-voiced chords as, for example, in the abovementioned Tonal Harmony Analysis app. Once students have mastered the harmonic principles and voice-leading rules, they can apply them more freely in a variety of compositional contexts - and not just in four-voice settings.

There are a number of chord symbol systems in use by musicians from different communities. In relation to the common-practice harmonic style - established in classical music but widely adopted by other traditions - the Roman numeral chord system was adopted, and app users can choose between the British and the American version. Both chord-symbol systems similarly embody key features that can be used to articulate the harmonic principles and voiceleading rules.

For programming purposes, the chord symbols are converted into a vector form: $\langle\mathrm{a}, \mathrm{b}, \mathrm{c}, \mathrm{d}\rangle$. This vector embodies salient features of each chord, namely, its key, scale degree, chord type and chord inversion. An earlier version of this vector system had been developed for a web-based programme [7], the present app builds on this.

\subsection{Validating the chord progression}

The set of declarative harmonic rules developed for the earlier web-based predecessor was also refined and expanded. These if-then rules express in programming terms the conventional harmonic principles and voice-leading rules. In addition, to foster more contextual understanding of harmonic progressions, the rules also factor in certain phrasestructural considerations such as standard tonal cadences (that is, conventional two-chord progressions used to end a phrase) and broader considerations in the use of mixture harmonies. At a more local level, the evaluation also considers threechord clichés such as those involving second inversion chords.

Given that the evaluative rules are formulated in accordance with conventional musical ones, it is therefore easy to attach specific feedback statements to them such that once a particular rule-violation occurs, the user can be automatically informed of the specific technical error. In general, through the feedback, users will learn about root movements in chord progressions, bass-movement in melodic terms, the need for harmonic resolutions, and other musicgrammatical considerations. 


\section{Designing the music-tutoring app}

\subsection{The basic user interface}

A user interface was designed to allow app-users to create chord progressions by conveniently dragging individual chord symbols from a panel of available chords into a placement box (see Figure 1).

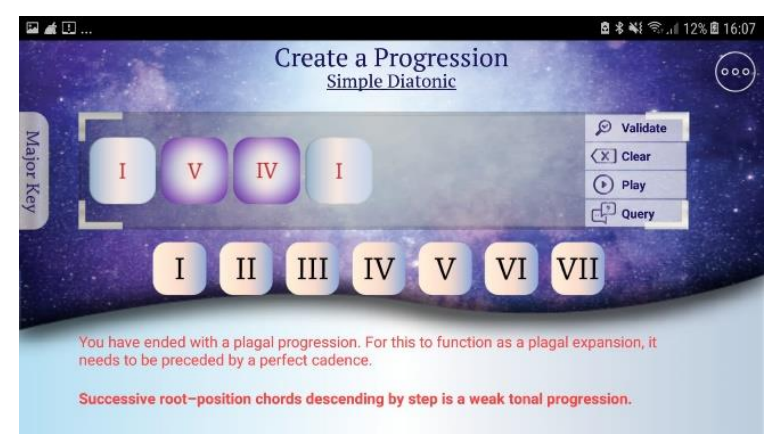

Figure 1. User interface of Harmonia-on-the-Go for creating and validating chord progression.

The user can then tap the "Play" button to hear the chord progression that has been created before tapping on the "Validate" button to have the progression evaluated by the app. Feedback statements are given in the space below the placement box. When a particular feedback statement is tapped, it appears in bold and the chord(s) in question are highlighted. In Figure 1 above, the highlighted error feedback refers to the undesirable V-IV progression. Having noted the error feedback, users can modify the progression and validate it again so as to eventually arrive at a grammatically sound progression.

\subsection{Progression in learning}

To guide the app user's learning, the app allows the user to move from the basic to the more advanced levels. This learning progression is mapped out into five levels of difficulty: (i) first steps, (ii) simple diatonic, (iii) advanced diatonic, (iv) mixture harmonies, and (v) applied dominants.

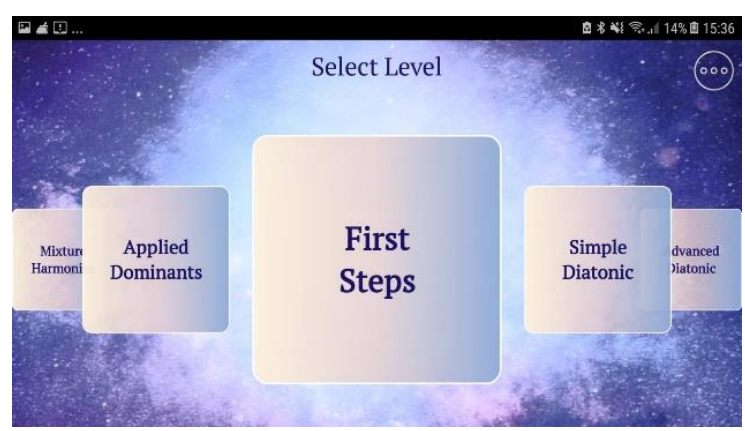

Figure 2. Selection of difficulty level from a carousel of options.
For each level, the user can choose to create the progression in either the major or minor key. The above learning sequence largely follows that found in most music theory textbooks on tonal harmony (e.g. $[8,9])$. Users however are free to move between levels in a non-linear fashion should they choose to do so.

\subsection{Learning analytics}

Good feedback gives learners clear guidance on how to improve, this knowledge is particularly useful for their self-regulated learning. To this end, the app offers three kinds of learning analytics if users have a registered account. First, they can track the percentage of total errors each day over a chosen period of time. Second, because the error-feedback statements have been classified into basic, intermediate and advanced levels, users can obtain a more detailed report of the errors they have made and how their learning has progressed over time. Finally, to be more targeted in their efforts to improve, they can also call up the top three most-frequent errors made each day so that they can tackle them more specifically.

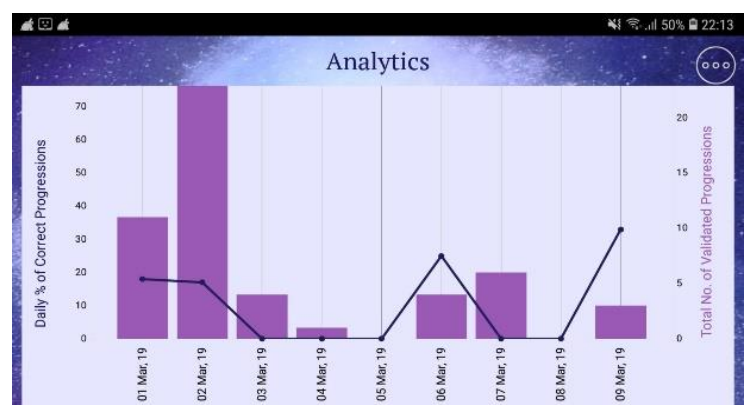

Figure 3. An example of learning analytics generated by the app.

For the teacher, the app's web console allows the monitoring of students' learning progress. The teacher not only has sight of the learning analytics that individual students have, he/she can also generate the learning analytics at the class or select group levels, thereby being able to identify any remedial needs of the students. Such insights can also inform future curriculum planning for improved delivery of the music theory contents.

\subsection{Interaction with the teacher}

For registered users, the app additionally allows them to pose questions should they wish to enquire about a particular error feedback, or should they want to make a more general enquiry. Figure 4 shows the user interface for this "Query" function. 


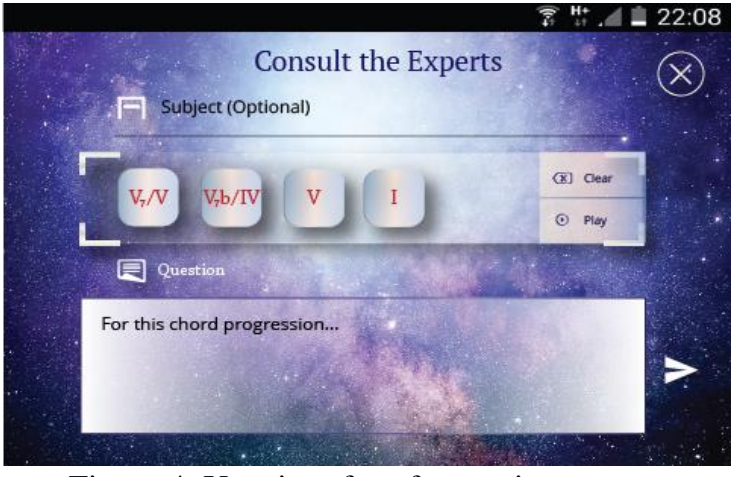

Figure 4. User interface for posting a query.

The "expert" - in this case, the teacher - will receive the query on the web console and a response can then be sent back to the individual user who will receive it via the app on their mobile. This app functionality allows for out-of-class asynchronous interaction between the teacher and individual students, thereby supplementing the automated feedback and learning analytics that the students have received.

\section{Pilot Test Findings}

\subsection{The Pilot Test}

The app was piloted with fifty students from four Secondary and one Junior College (equivalent to senior high). To prepare the participating teachers, a workshop was organised to familiarise them with the app. They were given (i) information about the music app, including the particular harmonic theory upon which the app was developed, (ii) some sample teaching ideas that involved the use of the app, and (iii) a list of music concepts that can be taught through the use of this app. They were however given the freedom to use the app in any way desired, in accordance to their particular school music curriculum. This was to minimize any disruption to their existing teaching plans. On average, the participating schools piloted the app for about five weeks.

Prior to the introduction of the app, the school students were given a pre-test to solicit information about their musical knowledge of harmony. At the end of the piloting period, a post-test was administered to see if there was any improvement in the students' knowledge of harmony as well as to understand their user experience of the app. Of the 50 students, only 37 completed the post-test and post-pilot survey. The participating teachers were also asked to provide their post-pilot feedback.

\subsection{Some findings}

The post-survey results were encouraging. $94.6 \%$ of the 37 respondents reported positive user experiences:
When using the app to create chord progressions, the feedback given by the app taught me how to better plan chord progressions that sound nice and how I can improve in my creation of chord progressions. (Student X)

It helped me understand harmonic phrase model and learn new chords. (Student Y)

It was fun to experiment and explore different chord progressions. (Student Z)

Most pertinently, 15 students (40.5\%) stated how they had benefited from the immediate feedback automated by the app when validating their created chord progressions

A comparison of the pre- and post-tests suggests that there was some positive learning outcome. For example, in both the pre-test and post-test, an erroneous chord progression was given. More students were able to identify and explain the errors in the post-test. The number of students who were only able to identify but not explain the errors decreased, likewise the number of students who made wrong diagnoses. The number of students who could not identify any errors at all dropped drastically between the pre- and post-test to a third of the initial 18. It is encouraging to note that in the post-test, 9 students made reference to the Harmonic Phrase Model or explained errors with phrases drawn from the App feedback statements.

The teachers' feedback was also generally positive. Besides appreciating the automated feedback, four of the teachers mentioned that the app enabled the students to conveniently experiment with creating chord progressions. The audio playback feature was particularly useful to students who do not play the piano.

\subsection{Limitations}

It is unfortunate that 13 out of the 50 students did not submit the post-test and post-survey. The overall use of the app based on the online user data collected was low, which probably explains the rather neutral response on the usefulness of the learner analytics. To be sure, one may understandably cast doubt on the positive self-report responses noted above. Nonetheless, the positive learning outcome, more objectively observed when comparing the pre- and post-test answers, supports some optimism here. A more systematic and extensive study is needed to better evaluate the efficacy of this music app as a personal tutoring tool. 


\section{Implications for the future}

Whilst the pilot test above has its limitations and its findings cannot be taken as conclusive, one may still recognize the attractive affordances of the app for the teaching of music theory in a mobile-learning age. For the digital generation used to the conveniences of digital and mobile technologies, and for whom learning on the move and instant feedback are of prime importance, this app can meet the challenge of for teaching music theory in the mobile digital age.

If well adopted by the teacher, it can be a powerful tool to encourage extensive self-practice - especially of the drill-and-practice kind - without unduly adding marking burden on the teacher. Just as significantly, the AI-enabled automated feedback and the learning analytics generated can go a long way to foster agency for self-regulated learning. It has been recognized that self-monitoring and control of one's own learning are important aspects of effective mobile learning [10]. Based on the learning analytics, students can choose the appropriate difficulty levels and select the relevant chords to be more targeted in their self-practice. On the other hand, should any student grasp the foundations faster than their peers, they may move on to more advanced levels on their own and still be "tutored" by the app, if not by the teacher via the app's "Query" function.

It should also be added that, for a subject like music, it is certainly an important benefit that the app can encourage music creativity even as the students master the music-grammar rules (recall Student Z's feedback). The playback function adds an audio feedback for the user who is experimenting with the chords.

In sum, Harmonia-on-the-Go opens up new pedagogical spaces for teaching music theory: it augments the traditional face-to-face classroom space and its pen-and-paper forms of practice; it empowers students to take more control of their learning. It is hoped that the convenience of mobile access will motivate students to practice more as well.

\section{Acknowledgements}

The development of the mobile app is funded by the Singapore Ministry of Education (Edulab project, AED 01/17 CKM). Assisting the author who is the PI are co-PIs Mr Tan Kheng Leong and Dr Cheng Yuan Shan, the collaborator from the ministry Mrs Ee-Chek Yui Hong, and research assistant Ms Ng Sock Poh.

\section{References}

[1] G. Lewis-Kraus, "The great AI awakening", The New York Times, 2016.
[2] B.P. Woolf, H.C. Lane, V.K. Chaudhri, and J.L. Kolodner, "AI Grand Challenges for Education", AI Magazine, 2013, pp. 66-86.

[3] E.D. Wagner, "Enabling Mobile Learning", EDUCAUSE Review, 2005, pp. 41-42.

[4] B. Eschenbrenner, and F.F.-H. Nah, "Mobile technology in education: uses and benefits", International Journal of Mobile Learning and Organisation, 2007, pp. 159-183.

[5] Sharples, M., J. Taylor, and G. Vavoula, "A theory of learning for the mobile age", in R. Andrews and C. Haythornthwaite (Eds.), The Sage Handbook of E-learning Research, Sage publications, London, 2007, pp. 221-245.

[6] H. Crompton, D. Burke, and K.H. Gregory, "The use of mobile learning in PK-12 education: A systematic review", Computers \& Education, 2017, pp. 51-63.

[7] E.K.M. Chong, E.K.M. and D. Qin, "Symbolic representation of chords for rule-based evaluation of tonal progressions", eContact!, 2014, available at https://econtact.ca/16_2/chong-ding_representation.html

[8] S. Laitz, The complete musician: An integrated approach to tonal theory, analysis, and listening, Oxford University Press, New York, 2003.

[9] M. Roig-Francolí, Harmony in context, McGraw-Hill, Boston, 2003.

[10] L. Sha, C.K. Looi, W. Chen, and B.H. Zhang, "Understanding mobile learning from the perspective of self-regulated learning", Journal of Computer Assisted Learning, 2012, pp. 366-378. 